\title{
molecules
}

ISSN 1420-3049

www.mdpi.com/journal/molecules

Article

\section{Synthesis and Biological Activity Evaluation of Novel $\beta$-Substituted Nitromethylene Neonicotinoid Analogues}

\section{Baozhu Wang, Jiagao Cheng, Zhiping Xu, Xiaoyong Xu, Xusheng Shao and Zhong Li *}

Shanghai Key Laboratory of Chemical Biology, School of Pharmacy, East China University of Science and Technology, P.O. Box 544, 130 Meilong Road, Shanghai 200237, China

* Author to whom correspondence should be addressed; E-Mail: lizhong@ecust.edu.cn;

Tel.: +86-021-6425-2603; Fax: +86-021-6425-3540.

Received: 25 June 2012; in revised form: 13 August 2012 / Accepted: 14 August 2012 /

Published: 24 August 2012

\begin{abstract}
The structure-based design and synthesis of a series of novel neonicotinoid analogues are described. The novel neonicotinoid analogues were designed based upon the reaction of enamine derivatives with electron-withdrawing $\beta$-substituents with electrophilic thiocyanogen reagents. These compounds were characterized by spectroscopic methods. Bioassays indicated that some of the synthesized compounds exhibited excellent bioactivity against cowpea aphids (Aphis craccivora). The $\mathrm{LC}_{50}$ values of compounds 7, 9, 12, 13, 15, 17, 19, 20 and commercial imidacloprid were 0.01567, 0.00974, 0.02494, $0.01893,0.02677,0.01778,0.0220,0.02447$ and $0.03502 \mathrm{mmol} \mathrm{L}^{-1}$, respectively, which suggested that they could be used as leads for future development of new insecticides.
\end{abstract}

Keywords: neonicotinoid analogues; new design strategy; thiocyanogen; insecticidal activities

\section{Introduction}

In recent years, neonicotinoid insecticides have been the fastest growing class of insecticides in modern crop protection, with widespread use against a broad spectrum of sucking and certain chewing pests [1-5]. Significant progress had been obtained on the chemistry of the insecticidal nicotinic agonists and the chemical biology of the nicotinic acetylcholine receptors (nAChRs), that is, chemorational approaches including physicochemical considerations, metabolism, resistance 
mechanisms, and chemical and structural biology aspects potentially expediting receptor structure-guided insecticide design [6-12].

Our past endeavors in this field have focused on systematic pharmacophore modifications of nitromethylene neonicotinoids that appeared to capture the "bioactive" conformation (Figure 1). Several types of nitromethylene neonicotinoids with the cis-configuration were presented; that is, neonicotinoids $\mathbf{1}$ with a tetrahydropyridine fixed cis-configurations; neonicotinoids $\mathbf{2}$ with bulky group fixed cis-configurations, neonicotinoids $\mathbf{3}$ with cis-configuration constructed by aza-Diels-Alder reactions, and divalent and oxa-bridged neonicotinoids 4 constructed from dialdehydes (Figure 1). Bioassays indicated that the synthesized cis compounds 1-4 exhibited excellent bioactivity against cowpea aphids [13-16]. According to our knowledge, the nitro groups in all commercialized neonicotinoids have a trans configuration, on which three proposals for mode of action are based [17]. The high bioactivity of compounds 1-4 implies that neonicotinoids in the cis configuration might bind to the receptor in a different way [13]. These efforts accordingly encourage the discovery of "superneonicotinoids" effective for imidacloprid-resistant pests and lepidoptera species.

Figure 1. Structures of imidacloprid neonicotinoids and structurally related analogues.<smiles>O=[N+]([O-])/N=C1\NCCN1Cc1ccc(Cl)nc1</smiles>

imidacloprid<smiles>O=[N+]([O-])/C(=C/c1ccco1)C1=NCCN1Cc1ccc(Cl)nc1</smiles>

2<smiles>O=[N+]([O-])C=C1NCCN1Cc1ccc(Cl)nc1</smiles>

nitromethylene neonicotinoid<smiles>[R]C1C(C#N)=C(N)N2CCN(Cc3ccc(Cl)nc3)C2=C1[N+](=O)[O-]</smiles>

3<smiles>[R7]OC1CC([R2])C([N+](=O)[O-])=C2N(Cc3ccc(Cl)nc3)CCN21</smiles>

1<smiles>O=[N+]([O-])C1=C2N(Cc3ccc(Cl)nc3)CCN2C2CCC1O2</smiles>

4

The structure derivative method through the organic reaction is another important tool for systematic pharmacophore modifications [18]. It had been theorized that the nitroenamine entities in these high active compounds play important roles for specific binding to the desired insect nAChRs site [19-22]. Encouraged by these reports, the modifications of nitroenamine entities to fix its configuration through organic reactions has attracted our attention. The chemistry of enamines, including push-pull enamines bearing an additional electron-acceptor group at the $\beta$-position, has been extensively developed, mainly as a result of electrophilic reactions that proceed predominantly at the $\beta$-position [23-25]. Electrophilic modification, followed by a set of transformations, have made enamines an integral part of synthetic organic chemistry methodology [26]. Additionally, electron-acceptor 
groups can participate in chemical transformations. According to this knowledge, a series of prototype compounds with extended N-substituted (thiocyanogen) substituents were designed in this paper (Figure 2). The choice of the thiocyanogen was predominantly governed by its traits as electrophilic reagent, and mainly by its ability to easily cyclize with the NH group in the imidazoline ring to fix the electron-withdrawing group in the cis position (Figure 2) [27].

Figure 2. Structures of imidacloprid neonicotinoids and its structurally related analogues.

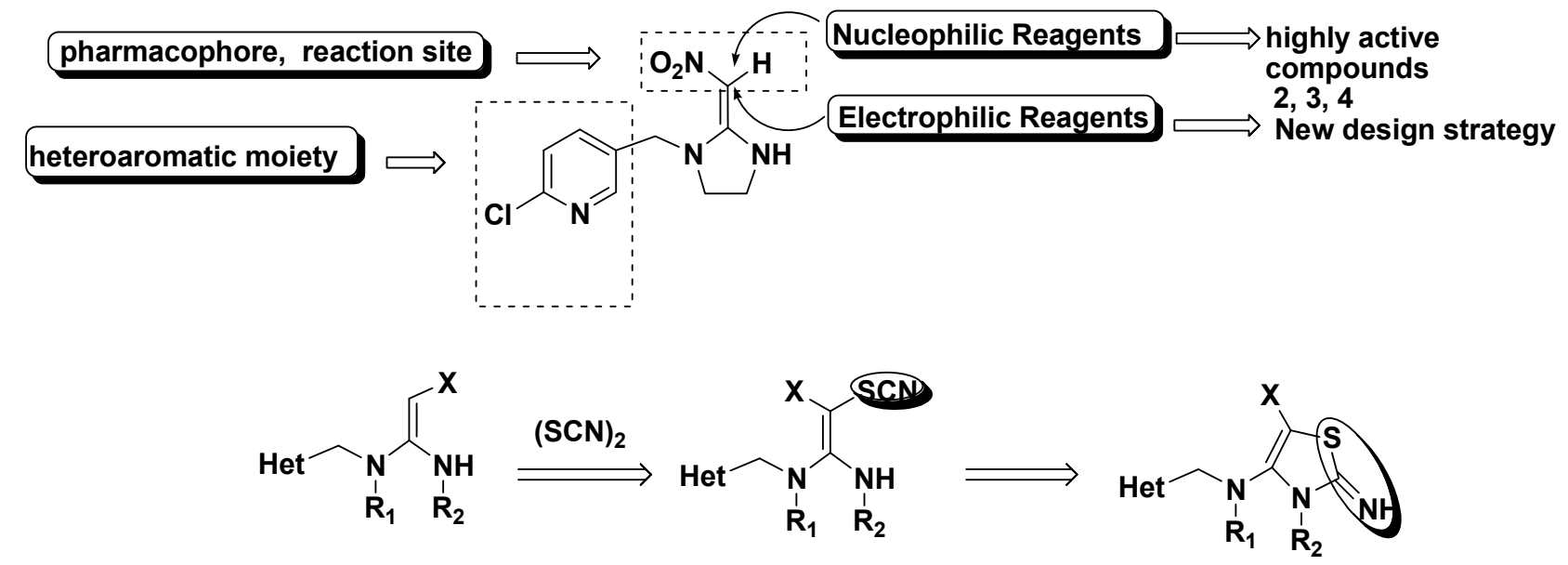

\section{Results and Discussion}

\subsection{Synthetic Chemistry}

The synthetic route of the target compounds is summarized in Scheme 1. The chemical structures of the novel neonicotinoid analogues under study are depicted in Figure 3. The preparation of the designed compounds began with the common intermediates $\mathbf{5 a}-\mathbf{h}$ and $\mathbf{6 a}-\mathbf{c}$ which were synthesized according to the method of Kagabu et al. [19]. In the reaction of $\mathbf{5 a}-\mathbf{h}$ with thiocyanogen at $0{ }^{\circ} \mathrm{C}$ for $1.5 \mathrm{~h}$, the corresponding $\beta$-thiocyanated products $7-14$ were produced in $63 \%-82 \%$ yields. In this procedure, 5a appears to produce a mixture of $\mathbf{7}$ and cyclization product $\mathbf{1 5}$. Similarly, $\mathbf{5 c}$ and $\mathbf{5 f}$ also gave mixtures of aminothiazole and the relevant cyclization product. Compounds 7, 9 and 12 were synthesized by reducing the reaction time and reaction temperature without forming cyclization products. Then, these compounds 7-9 and 11-13 were converted quantitatively into the corresponding cyclization products, 15-20, by stirring at room temperature in dichloromethane solution containing $0.1 \%$ piperidine as catalyst. Unexpectedly, $\mathbf{1 0}$ and $\mathbf{1 4}$ could not be converted into the corresponding cyclization products. The substrates $\mathbf{6 a}-\mathbf{c}$ yielded only the corresponding cyclization products 21-23 respectively under the same conditions. Whereas configuration of compounds 7-14 and 21-23 cannot be determined easily by standard ${ }^{1} \mathrm{H}$ - and ${ }^{13} \mathrm{C}$-NMR techniques, the compounds $\mathbf{1 5}-\mathbf{2 0}$ obtained from the reaction system are considered to be produced with retention of the configuration of the preformed enamines with electron-withdrawing $\beta$-substituents, followed by catalyzed cyclization. The structures of the title compounds were well characterized by ${ }^{1} \mathrm{H}-\mathrm{NMR},{ }^{13} \mathrm{C}-\mathrm{NMR}$ and HRMS. 
Scheme 1. Synthetic route for the title compounds 7-23.<smiles>[X]/C=C1\NCN(C[R])C1C[R]</smiles>

$5 a-h$

$5 a$

$5 b$

$5 c$

$5 d$

$5 e$

$5 f$

$5 \mathrm{~g}$

$5 \mathrm{~h}$

$\begin{array}{llll}\mathrm{X} & \mathrm{NO} & \mathrm{CN} \\ \mathrm{R}_{1}-\mathrm{CH}_{2} \mathrm{CH}_{2-} & -\mathrm{CH}_{2} \mathrm{CH}_{2}^{-} & -\mathrm{CH}_{2} \mathrm{CH}_{2-} \\ \end{array}$

CN

$\mathrm{NO}_{2}$

$\mathrm{NO}_{2}$

$\mathrm{NO}_{2}$

$\mathrm{NO}_{2}$<smiles>[R4]N/C(=C/[N+](=O)[O-])N([R3])Cc1ccc(Cl)nc1</smiles><smiles>Cc1cnc(Cl)s1</smiles><smiles>Cc1ccccc1</smiles><smiles>Cc1ccc(Cl)nc1</smiles><smiles>Cc1ccc(Cl)nc1</smiles>

6a-c

$$
\begin{array}{ll}
\text { 6a } \mathrm{R}_{3}=\mathrm{CH}_{3} \mathrm{CH}_{2}- & \mathrm{R}_{4}=\mathrm{CH}_{3}- \\
\text { 6b } \mathrm{R}_{3}=\mathrm{H} & \mathrm{R}_{4}=\mathrm{CH}_{3}- \\
\text { 6c } \mathrm{R}_{3}=\mathrm{H} & \mathrm{R}_{4}=\mathrm{CH}_{3} \mathrm{CH}_{2}-
\end{array}
$$

Figure 3. Chemical structures of novel neonicotinoid analogues under study.<smiles>[X]C(S#N)=C1NCCN1Cc1ccc(Cl)nc1</smiles><smiles>[X]C(S)=C1NCCN1Cc1cnc(Cl)s1</smiles><smiles>N#S/C(=C1\NCCN1Cc1ccccc1)[N+](=O)[O-]</smiles>

$$
\begin{aligned}
& 7 \mathrm{X}=\mathrm{NO}_{2} \\
& 8 \mathrm{X}=\mathrm{CN}
\end{aligned}
$$


Figure 3. Cont.<smiles>N#CSC(C#N)=C1NCCCN1Cc1ccc(Cl)nc1</smiles>

12<smiles>[X]c1sc(=N)n2c1N(Cc1ccc(Cl)nc1)CC2</smiles>

$15 \mathrm{X}=\mathrm{NO}_{2}$

$16 \mathrm{X}=\mathrm{CN}$<smiles>N=c1sc([N+](=O)[O-])c2n1CCCN2Cc1ccc(Cl)nc1</smiles>

19<smiles>N#CC(SC1NCCN1Cc1ccc(C(F)(F)F)nc1)=C([N+](=O)[O-])[N+](=O)[O-]</smiles>

13<smiles>N=c1sc([N+](=O)[O-])c2n1CCN2Cc1cnc(Cl)s1</smiles>

17<smiles>N=c1sc([N+](=O)[O-])c2n1CCN2Cc1ccc(C(F)(F)F)nc1</smiles>

20<smiles>N#CC(C#N)=C1Nc2ccccc2N1Cc1ccc(Cl)nc1</smiles>

14<smiles>N=c1sc([N+](=O)[O-])c2n1CCN2Cc1ccccc1</smiles>

18<smiles>[R]N(Cc1ccc(Cl)nc1)c1c([N+](=O)[O-])sc(=N)n1[R]</smiles>

$$
\begin{array}{lll}
21 & \mathrm{R}_{1}=\mathrm{CH}_{3} \mathrm{CH}_{2}- & \mathrm{R}_{2}=\mathrm{CH}_{3^{-}} \\
22 & \mathrm{R}_{1}=\mathrm{H} & \mathrm{R}_{2}=\mathrm{CH}_{3^{-}} \\
23 & \mathrm{R}_{1}=\mathrm{H} & \mathrm{R}_{2}=\mathrm{CH}_{3} \mathrm{CH}_{2}^{-}
\end{array}
$$

\subsection{Biological Activities}

The insecticidal activities of the designed compounds against cowpea aphids are shown in Table 1. Most of the title compounds showed excellent insecticidal activities against cowpea aphids. For example, the $\mathrm{LC}_{50}$ values of compounds 7, 9, 12, 13, 15, 17, 19, and 20 were 0.01567, 0.00974, $0.02494,0.01893,0.02677,0.01778,0.0220$ and $0.02447 \mathrm{mmol} \mathrm{L}^{-1}$, respectively, whereas that of imidacloprid was only $0.03502 \mathrm{mmol} \mathrm{L}^{-1}$ [16]. Activities varied significantly depending upon the patterns of substituents on the enamine and imidazole ring. It was observed that compounds with $\mathrm{NO}_{2}$ substituents $(7,15,9$ and 17) demonstrated better activities than that with $\mathrm{CN}$ substituents $(\mathbf{8}, 10$ and 16). Generally, the imidazolidine substituents appear to be the important factor for insecticidal activities. A benzene substituent (compound 14) resulted in complete inactivity. Moreover, the cyclic addition of SCN substituents with the imidazolidine ring is unfavourable for high activities from the data analysis present of compounds 7, 9, 15 and 17. 
Table 1. Insecticidal activities of compounds 7-23 against cowpea aphids (Aphis craccivora).

\begin{tabular}{|c|c|c|}
\hline Compounds & Mortality $\left(\%, 500 \mathrm{mg} \mathrm{L}^{-1}\right)$ & $\mathrm{LC}_{50}\left(\mathrm{mmol} \mathrm{L}^{-1}\right)$ \\
\hline 7 & 100 & 0.01567 \\
\hline 8 & 34.6 & $\mathrm{nt}^{\mathrm{a}}$ \\
\hline 9 & 100 & 0.00974 \\
\hline 10 & 0 & $\mathrm{nt}$ \\
\hline 11 & 23.98 & nt \\
\hline 12 & 100 & 0.02494 \\
\hline 13 & 100 & 0.01893 \\
\hline 14 & 87.82 & $\mathrm{nt}$ \\
\hline 15 & 100 & 0.02677 \\
\hline 16 & 32.4 & $\mathrm{nt}$ \\
\hline 17 & 100 & 0.01778 \\
\hline 18 & 16.19 & $\mathrm{nt}$ \\
\hline 19 & 100 & 0.02200 \\
\hline 20 & 100 & 0.02447 \\
\hline 21 & 57.6 & $\mathrm{nt}$ \\
\hline 22 & 100 & 0.11839 \\
\hline 23 & 50.79 & $\mathrm{nt}$ \\
\hline
\end{tabular}

\section{Experimental}

\subsection{General}

Melting points (mp) were recorded on a Büchi B540 apparatus (Büchi Labortechnik AG, Flawil, Switzerland) and are uncorrected. ${ }^{1} \mathrm{H}-\mathrm{NMR}$ and ${ }^{13} \mathrm{C}$-NMR spectra were recorded on a Bruker AM-400 (400 MHz) spectrometer with $\mathrm{CDCl}_{3}$ or DMSO- $d_{6}$ as the solvent and TMS as the internal standard. Chemical shifts are reported in $\delta$ (parts per million) values. High-resolution mass spectra were recorded under electron impact $(70 \mathrm{eV})$ condition using a MicroMass GCT CA 055 instrument. Analytical thin-layer chromatography (TLC) was carried out on precoated plates (silica gel $60 \mathrm{~F}_{254}$ ), and spots were visualized with ultraviolet (UV) light. The compounds $\mathbf{5 a}-\mathbf{h}$ and $\mathbf{6 a}-\mathbf{c}$ were synthesized according to the method of Kagabu et al. [19]. Unless otherwise noted, reagents and solvents were used as received from commercial suppliers. Yields were not optimized. All reactions were carried out under a protective atmosphere of drying nitrogen or utilizing a calcium chloride drying tube.

\subsubsection{General Synthetic Procedure for 7-14}

To a solution of $\mathbf{5 a}-\mathbf{g}(10 \mathrm{mmol})$ in dichloromethane $(100 \mathrm{~mL})$, a thiocyanogen $(10 \mathrm{mmol})$ solution in dichloromethane $(50 \mathrm{~mL})$, which was previously prepared from lead thiocyanate and bromine at $-15{ }^{\circ} \mathrm{C}$, was added dropwise with stirring at $-15{ }^{\circ} \mathrm{C}$ and then the mixture was stirred for $1.5 \mathrm{~h}$ at $0{ }^{\circ} \mathrm{C}$. The reaction mixture was washed with cold water $(3 \times 50 \mathrm{~mL})$ and brine $(50 \mathrm{~mL})$, and dried with sodium sulfate. The solvent was removed to give a crude solid, which was recrystallized from a mixture of dichloromethane/ether $(2: 1, \mathrm{v} / \mathrm{v})$ to give 7-14. 
2-Chloro-5-((2-(nitro(thiocyanato)methylene)imidazolidin-1-yl)methyl)pyridine (7). Yield: 92\%, m.p.: 130.6-132.1 ${ }^{\circ} \mathrm{C} ;{ }^{1} \mathrm{H}-\mathrm{NMR}$ (DMSO- $d_{6}$ ): $\delta 3.76$ (br.s, 4H), 4.77 (s, 2H), 7.55 (d, $\left.J=8.4 \mathrm{~Hz}, 1 \mathrm{H}\right), 7.86$ $\left(\mathrm{dd}, J_{l}=2.6 \mathrm{~Hz}, J_{2}=8.4 \mathrm{~Hz}, 1 \mathrm{H}\right), 8.42(\mathrm{~d}, J=2.0 \mathrm{~Hz}, 1 \mathrm{H}), 9.43(\mathrm{~s}, 1 \mathrm{H}) \mathrm{ppm} ;{ }^{13} \mathrm{C}-\mathrm{NMR}$ (DMSO- $\left.d_{6}\right)$ : $\delta$ 162.6, 149.9, 149.1, 139.1, 131.8, 124.7, 112.3, 87.5, 50.7, 50.2, 42.7; HRMS (ES+) calcd for $\mathrm{C}_{11} \mathrm{H}_{10}{ }^{35} \mathrm{ClN}_{5} \mathrm{O}_{2} \mathrm{~S}(\mathrm{M}+\mathrm{H})^{+}: 312.0244$. Found: 312.0330 . Calcd for $\mathrm{C}_{11} \mathrm{H}_{10}{ }^{37} \mathrm{ClN}_{5} \mathrm{O}_{2} \mathrm{~S}(\mathrm{M}+\mathrm{H})^{+}: 314.0214$ Found: 314.0284 .

2-(1-((6-Chloropyridin-3-yl)methyl)imidazolidin-2-ylidene)-2-thiocyanatoacetonitrile (8). Yield: 63\%, m.p.: $118.2-119.7{ }^{\circ} \mathrm{C} ;{ }^{1} \mathrm{H}-\mathrm{NMR}$ (DMSO- $\left.d_{6}\right): \delta 3.51-3.56(\mathrm{~m}, 2 \mathrm{H}), 3.66-3.70(\mathrm{~m}, 2 \mathrm{H}), 4.79(\mathrm{~s}, 2 \mathrm{H})$, $7.54(\mathrm{~d}, J=8.4 \mathrm{~Hz}, 1 \mathrm{H}), 7.78-7.80(\mathrm{~m}, 1 \mathrm{H}), 8.11(\mathrm{~s}, 1 \mathrm{H}), 8.36(\mathrm{~d}, J=2.4 \mathrm{~Hz}, 1 \mathrm{H}) \mathrm{ppm} ;{ }^{13} \mathrm{C}-\mathrm{NMR}$ (DMSO-d $d_{6}$ : $\delta 164.9,150.0,149.2,139.2,132.1,124.8,122.1,113.8,51.6,47.4,41.5,31.2$; HRMS (EI+) calcd for $\mathrm{C}_{12} \mathrm{H}_{10}{ }^{35} \mathrm{ClN}_{5} \mathrm{~S}\left(\mathrm{M}^{+}\right)$: 291.0345. Found: 291.0344. Calcd for $\mathrm{C}_{12} \mathrm{H}_{10}{ }^{37} \mathrm{ClN}_{5} \mathrm{~S}\left(\mathrm{M}^{+}\right)$: 293.0316 Found: 293.0318.

1-((2-Chlorothiazol-5-yl)methyl)-2-(nitro(thiocyanato)methylene)imidazolidine (9). Yield: 75\%, m.p.: 85.1-86.3 ${ }^{\circ} \mathrm{C}$; ${ }^{1} \mathrm{H}-\mathrm{NMR}$ (DMSO- $\left.d_{6}\right): \delta 3.69-3.78(\mathrm{~m}, 4 \mathrm{H}), 4.84(\mathrm{~s}, 2 \mathrm{H}), 7.73(\mathrm{~s}, 1 \mathrm{H}), 9.40$ (s, 1H) ppm; ${ }^{13} \mathrm{C}-\mathrm{NMR}$ (DMSO- $\left.d_{6}\right): \delta 162.2,151.6,141.8,135.9,112.1,87.3,50.1,46.3,42.6$; HRMS (ES+) calcd for $\mathrm{C}_{9} \mathrm{H}_{8}{ }^{35} \mathrm{ClN}_{5} \mathrm{O}_{2} \mathrm{~S}_{2}(\mathrm{M}+\mathrm{H})^{+}$: 317.9808 . Found: 317.9890 . Calcd for $\mathrm{C}_{9} \mathrm{H}_{8}{ }^{37} \mathrm{ClN}_{5} \mathrm{O}_{2} \mathrm{~S}_{2}(\mathrm{M}+\mathrm{H})^{+}$: 319.9778 Found: 319.9860.

2-(1-((2-Chlorothiazol-5-yl)methyl)imidazolidin-2-ylidene)-2-thiocyanatoacetonitrile (10). Yield: 52\%, m.p.: $120.3-122.2{ }^{\circ} \mathrm{C} ;{ }^{1} \mathrm{H}-\mathrm{NMR}$ (DMSO- $d_{6}$ ): $\delta 3.30-3.70(\mathrm{~m}, 4 \mathrm{H}), 4.93$ (s, 2H), $7.70(\mathrm{~s}, 1 \mathrm{H}), 8.17$ (s, $1 \mathrm{H}) \mathrm{ppm} ;{ }^{13} \mathrm{C}-\mathrm{NMR}\left(\mathrm{DMSO}-d_{6}\right): \delta 164.5,151.3,141.6,135.8,122.0,113.5,51.0,43.0,41.5,31.8$; HRMS (ES-) calcd for $\mathrm{C}_{10} \mathrm{H}_{8}{ }^{35} \mathrm{ClN}_{5} \mathrm{~S}(\mathrm{M}-\mathrm{H})^{+}:$295.9910. Found: 295.9829. Calcd for $\mathrm{C}_{10} \mathrm{H}_{8}{ }^{37} \mathrm{ClN}_{5} \mathrm{~S}$ $(\mathrm{M}-\mathrm{H})^{+}: 297.9880$ Found: 297.9803.

1-Benzyl-2-(nitro(thiocyanato)methylene)imidazolidine (11). Yield: 82\%, m.p.: 102.3-104.2 ${ }^{\circ} \mathrm{C}$; ${ }^{1} \mathrm{H}-\mathrm{NMR}$ (DMSO- $\left.d_{6}\right): \delta 3.72(\mathrm{~s}, 4 \mathrm{H}), 4.75(\mathrm{~s}, 2 \mathrm{H}), 7.32-7.42(\mathrm{~m}, 5 \mathrm{H}), 9.40(\mathrm{~s}, 1 \mathrm{H}) \mathrm{ppm} ;{ }^{13} \mathrm{C}-\mathrm{NMR}$ (DMSO- $d_{6}$ ): $\delta 162.3,136.1,129.2,128.1,127.6,112.3,86.9,52.7,50.3,42.6$; HRMS (ES+) calcd for $\mathrm{C}_{12} \mathrm{H}_{12} \mathrm{~N}_{4} \mathrm{O}_{2} \mathrm{~S}(\mathrm{M}+\mathrm{H})^{+}:$277.0681. Found: 277.0750 .

1-((6-Chloropyridin-3-yl)methyl)-2-(nitro(thiocyanato)methylene)-hexahydropyrimidine (12). Yield: 70\%, m.p.: 158.1-160.3 ${ }^{\circ} \mathrm{C} ;{ }^{1} \mathrm{H}-\mathrm{NMR}$ (DMSO-d $)$ ): $\delta 2.14$ (t, $\left.J=5.2,2 \mathrm{H}\right), 3.38(\mathrm{t}, J=5.6 \mathrm{~Hz}, 2 \mathrm{H}$ ), $3.85(\mathrm{t}, J=5.6 \mathrm{~Hz}, 2 \mathrm{H}), 4.06(\mathrm{~s}, 2 \mathrm{H}), 7.57(\mathrm{~d}, J=8.0 \mathrm{~Hz}, 1 \mathrm{H}), 7.91\left(\mathrm{dd}, J_{1}=8.4 \mathrm{~Hz}, J_{2}=2.8 \mathrm{~Hz}, 1 \mathrm{H}\right)$, $8.45(\mathrm{~d}, J=2.4 \mathrm{~Hz}, 1 \mathrm{H}) \mathrm{ppm} ;{ }^{13} \mathrm{C}-\mathrm{NMR}$ (DMSO- $\left.d_{6}\right): \delta 164.7,158.9,150.2,146.2,140.1,131.0,124.5$, 106.5, 56.8, 47.2, 45.1, 19.1; HRMS (ES+) calcd for $\mathrm{C}_{12} \mathrm{H}_{12}{ }^{35} \mathrm{ClN}_{5} \mathrm{O}_{2} \mathrm{~S}(\mathrm{M}+\mathrm{H})^{+}:$326.0400. Found: 326.0477. Calcd for $\mathrm{C}_{12} \mathrm{H}_{12}{ }^{37} \mathrm{ClN}_{5} \mathrm{O}_{2} \mathrm{~S}(\mathrm{M}+\mathrm{H})^{+}$: 328.0371 Found: 328.0465 .

5-((2-(Nitro(thiocyanato)methylene)imidazolidin-1-yl)methyl)-2-(trifluoromethyl)pyridine (13). Yield: 82\%, m.p.: 140.1-142.3 ${ }^{\circ} \mathrm{C}$; ${ }^{1} \mathrm{H}-\mathrm{NMR}$ (DMSO-d $)$ ): $\delta 3.76$ (s, 4H), 3.38 (t, $J=5.6 \mathrm{~Hz}, 2 \mathrm{H}$ ), 4.85 $(\mathrm{s}, 2 \mathrm{H}), 7.91(\mathrm{~d}, J=8.0 \mathrm{~Hz}, 1 \mathrm{H}), 8.04(\mathrm{~s}, 1 \mathrm{H}), 8.75(\mathrm{~s}, 1 \mathrm{H}), 9.45(\mathrm{~s}, 1 \mathrm{H}) \mathrm{ppm} ;{ }^{13} \mathrm{C}-\mathrm{NMR}\left(\mathrm{DMSO}-d_{6}\right)$ : $\delta$ 162.7, 149.3, 137.2, 136.6, 121.1, 112.3, 87.7, 50.9, 50.8, 42.8; HRMS (ES+) calcd for $\mathrm{C}_{12} \mathrm{H}_{10} \mathrm{~F}_{3} \mathrm{~N}_{5} \mathrm{O}_{2} \mathrm{~S}$ $(\mathrm{M}+\mathrm{H})^{+}:$345.0507. Found: 345.0617 . 
(E-1-((6-Chloropyridin-3-yl)methyl)-2-(nitro(thiocyanato)methylene)-2,3-dihydro-1H-enzo[d] imid-azole (14). Yield: 74\%, m.p.: 125.6-127.2 ${ }^{\circ} \mathrm{C} ;{ }^{1} \mathrm{H}-\mathrm{NMR}$ (DMSO- $\left.d_{6}\right): \delta 4.79$ (s, 2H), 7.39-7.7 (m, 6H), 8.35 $(\mathrm{d}, J=2.4 \mathrm{~Hz}, 1 \mathrm{H}) \mathrm{ppm} ;{ }^{13} \mathrm{C}-\mathrm{NMR}\left(\mathrm{DMSO}-d_{6}\right): \delta 150.1,148.7,148.6,138.5,132.5,131.3,130.4$, 126.0, 125.4, 124.8, 113.8, 112.2, 112.0, 86.0, 55.4, 47.2; HRMS (ES-) calcd for $\mathrm{C}_{10} \mathrm{H}_{8}{ }^{35} \mathrm{ClN}_{5} \mathrm{~S}$ $(\mathrm{M}-\mathrm{H})^{+}:$358.0244. Found: 358.0130. Calcd for $\mathrm{C}_{10} \mathrm{H}_{8}{ }^{37} \mathrm{ClN}_{5} \mathrm{~S}(\mathrm{M}-\mathrm{H})^{+}: 360.0214$. Found: 297.0166.

\subsubsection{General Synthetic Procedure for 15-20}

The solution of 7-9 and 11-13 (10 mmol) in dichloromethane (50 mL) containing $0.1 \%$ piperidine was stirred for $2 \mathrm{~h}$ at room temperature. After removal of the solvent, the residue was recrystallized from dichloromethane to give $\mathbf{1 5 - 2 0}$ in quantitative yields.

1-((6-Chloropyridin-3-yl)methyl)-7-nitro-2,3-dihydroimidazo[1,2-c]thiazol-5(1H)-imine (15). Yield: 93\%, m.p.: $136.4-138.5{ }^{\circ} \mathrm{C} ;{ }^{1} \mathrm{H}-\mathrm{NMR}$ (DMSO-d $)$ ): $\delta 3.85$ (s, 2H), 4.08 (s, 2H), 5.27 (s, 2H), 7.58 (br.s, 1H), 7.91-7.92 (m, 1H), 8.47 (s, 1H), 8.91 (br.s, 1H) ppm; ${ }^{13} \mathrm{C}-\mathrm{NMR}$ (DMSO-d $)$ ): $\delta 150.6,150.1$, 149.7, 139.8, 132.0, 124.8, 99.7, 55.7, 49.4, 41.8; HRMS (ES+) calcd for $\mathrm{C}_{11} \mathrm{H}_{10}{ }^{35} \mathrm{ClN}_{5} \mathrm{O}_{2} \mathrm{~S}(\mathrm{M}+\mathrm{H})^{+}$: 312.0244. Found: 312.0321 . Calcd for $\mathrm{C}_{11} \mathrm{H}_{10}{ }^{37} \mathrm{ClN}_{5} \mathrm{O}_{2} \mathrm{~S}(\mathrm{M}+\mathrm{H})^{+}: 314.0279$. Found: 314.0284 .

1-((6-Chloropyridin-3-yl)methyl)-5-imino-1,2,3,5-tetrahydroimidazo[1,2-c]thiazole-7-carbonitrile (16). Yield: 82\%, m.p.: 201.5-202.3 ${ }^{\circ} \mathrm{C}$; ${ }^{1} \mathrm{H}-\mathrm{NMR}\left(\mathrm{DMSO}-d_{6}\right): \delta 3.87(\mathrm{~s}, 4 \mathrm{H}), 4.61(\mathrm{~s}, 2 \mathrm{H}), 7.41(\mathrm{~d}, J=8.0 \mathrm{~Hz}$, $1 \mathrm{H}), 7.73-7.76(\mathrm{~m}, 1 \mathrm{H}), 8.38(\mathrm{~d}, J=2.0 \mathrm{~Hz}, 1 \mathrm{H}) \mathrm{ppm} ;{ }^{13} \mathrm{C}-\mathrm{NMR}$ (DMSO- $\left.d_{6}\right): \delta 155.2,153.0,152.0$, 149.3, 138.9, 129.1, 125.0, 114.7, 53.2, 47.8, 47.2, 41.0; HRMS (ES+) calcd for $\mathrm{C}_{12} \mathrm{H}_{10}{ }^{35} \mathrm{ClN}_{5} \mathrm{~S}$ $(\mathrm{M}+\mathrm{H})^{+}:$292.0345. Found: 292.0430 . Calcd for $\mathrm{C}_{12} \mathrm{H}_{10}{ }^{37} \mathrm{ClN}_{5} \mathrm{~S}(\mathrm{M}+\mathrm{H})^{+}: 294.0316$. Found: 294.0410 .

1-((2-Chlorothiazol-5-yl)methyl)-7-nitro-2,3-dihydroimidazo[1,2-c]thiazol-5(1H)-imine (17). Yield: 78\%, m.p.: 150.3-151.4 ${ }^{\circ} \mathrm{C} ;{ }^{1} \mathrm{H}-\mathrm{NMR}$ (DMSO- $\left.d_{6}\right): \delta 3.81-4.16(\mathrm{~m}, 4 \mathrm{H}), 5.34(\mathrm{~s}, 2 \mathrm{H}), 7.77(\mathrm{~s}, 1 \mathrm{H})$, $8.96(\mathrm{~s}, 1 \mathrm{H}) \mathrm{ppm} ;{ }^{13} \mathrm{C}-\mathrm{NMR}\left(\mathrm{DMSO}-d_{6}\right): \delta 152.3,150.0,142.0,136.0,99.9,55.5,45.2,41.7$; HRMS $(\mathrm{EI}+)$ calcd for $\mathrm{C}_{9} \mathrm{H}_{8} \mathrm{ClN}_{5} \mathrm{O}_{2} \mathrm{~S}_{2}\left(\mathrm{M}^{+}\right)$: 316.9808 . Found: 316.9807 .

1-Benzyl-7-nitro-2,3-dihydroimidazo[1,2-c]thiazol-5(1H)-imine (18). Yield: 74\%, m.p.: 82.3-84.4 ${ }^{\circ} \mathrm{C}$; ${ }^{1} \mathrm{H}-\mathrm{NMR}\left(\mathrm{DMSO}-d_{6}\right): \delta$ 3.81-4.06 (m, 4H), $5.26(\mathrm{~s}, 2 \mathrm{H}), 7.32-7.43(\mathrm{~s}, 1 \mathrm{H}), 8.96(\mathrm{~s}, 1 \mathrm{H}) \mathrm{ppm}$; ${ }^{13} \mathrm{C}-\mathrm{NMR}$ (DMSO- $\left.d_{6}\right): \delta 150.2,136.4,129.2,128.3,55.5,52.3,41.6$; HRMS (ES+) calcd for calcd for $\mathrm{C}_{12} \mathrm{H}_{12} \mathrm{~N}_{4} \mathrm{O}_{2} \mathrm{~S}(\mathrm{M}+\mathrm{H})^{+}:$277.0681. Found: 277.0746.

1-((6-Chloropyridin-3-yl)methyl)-8-nitro-1,2,3,4-tetrahydrothiazolo[3,4-a]pyrimidin-6-imine

Yield: 65\%, m.p.: $165.3-167.4{ }^{\circ} \mathrm{C}$; ${ }^{1} \mathrm{H}-\mathrm{NMR}$ (DMSO- $\left.d_{6}\right): \delta 2.01(\mathrm{t}, J=5.2 \mathrm{~Hz}, 2 \mathrm{H}), 3.30(\mathrm{t}, J=5.6 \mathrm{~Hz}$, $2 \mathrm{H}), 3.73(\mathrm{t}, J=5.6 \mathrm{~Hz}, 2 \mathrm{H}), 4.90(\mathrm{~s}, 2 \mathrm{H}), 7.53(\mathrm{~d}, J=8.4 \mathrm{~Hz}, 1 \mathrm{H}), 7.92\left(\mathrm{dd}, J_{l}=8.4 \mathrm{~Hz}, J_{2}=2.4 \mathrm{~Hz}\right.$, $1 \mathrm{H}), 8.43(\mathrm{~d}, J=2.0,1 \mathrm{H}), 9.19(\mathrm{~s}, 1 \mathrm{H}) \mathrm{ppm} ;{ }^{13} \mathrm{C}-\mathrm{NMR}\left(\mathrm{DMSO}-d_{6}\right): \delta 153.5,150.5,150.0,149.8$, 139.9, 131.9, 124.4, 103.2, 56.5, 47.3, 41.6, 19.3; HRMS (ES+) calcd for $\mathrm{C}_{12} \mathrm{H}_{12}{ }^{35} \mathrm{ClN}_{5} \mathrm{O}_{2} \mathrm{~S}(\mathrm{M}+\mathrm{H})^{+}$: 326.0400. Found: 326.0476 . Calcd for $\mathrm{C}_{12} \mathrm{H}_{12}{ }^{37} \mathrm{ClN}_{5} \mathrm{O}_{2} \mathrm{~S}(\mathrm{M}+\mathrm{H})^{+}: 328.0371$. Found: 328.0473 .

7-Nitro-1-((6-(trifluoromethyl)pyridin-3-yl)methyl)-2,3-dihydroimidazo[1,2-c]thiazol-5(1H)-imine (20). Yield: 88\%, m.p.: $148.2-149.3{ }^{\circ} \mathrm{C}$; ${ }^{1} \mathrm{H}-\mathrm{NMR}\left(\mathrm{DMSO}-d_{6}\right): \delta 3.85(\mathrm{t}, J=8.2 \mathrm{~Hz}, 2 \mathrm{H}), 4.10(\mathrm{t}, J=9.2 \mathrm{~Hz}$, 2H), $5.36(\mathrm{~s}, 2 \mathrm{H}), 7.94(\mathrm{~d}, J=8.4 \mathrm{~Hz}, 1 \mathrm{H}), 8.13(\mathrm{~d}, J=8.4 \mathrm{~Hz}, 1 \mathrm{H}), 8.81(\mathrm{~s}, 1 \mathrm{H}), 8.94$ (s, 1H) ppm; 
${ }^{13} \mathrm{C}-\mathrm{NMR}\left(\mathrm{DMSO}-d_{6}\right): \delta$ 151.0, 149.8, 137.8, 136.8, 121.1, 56.0, 50.0, 41.8; HRMS (ES+) calcd for $\mathrm{C}_{12} \mathrm{H}_{10} \mathrm{~F}_{3} \mathrm{~N}_{5} \mathrm{O}_{2} \mathrm{~S}(\mathrm{M}+\mathrm{H})^{+}: 346.0507$. Found: 346.0634 .

\subsubsection{General Synthetic Procedure for 21-23}

To a solution of $\mathbf{6 a}-\mathbf{c}(10 \mathrm{mmol})$ in dichloromethane $(50 \mathrm{~mL})$ was added with stirring at $-15{ }^{\circ} \mathrm{C}$ a dichloromethane solution of thiocyanogen $(10 \mathrm{mmol})$ which had previously prepared from lead thiocyanate and bromine at $-15^{\circ} \mathrm{C}$, and then the mixture was stirred for $1.5 \mathrm{~h}$ at room temperature. The reaction mixture was washed with cold water $(3 \times 50 \mathrm{~mL})$ and brine $(50 \mathrm{~mL})$ and then dried with sodium sulfate. The solvent was evaporated in vacuo, and fractional precipitation of the residue from dichloromethane afforded 21-23.

$N$-((6-Chloropyridin-3-yl)methyl)-N-ethyl-2-imino-3-methyl-5-nitro-2,3-dihydrothiazol-4-amine (21). Yield: $84 \%$, m.p.: $120.3-121.4{ }^{\circ} \mathrm{C}$; ${ }^{1} \mathrm{H}-\mathrm{NMR}$ (DMSO- $\left.d_{6}\right): \delta 1.14(\mathrm{t}, J=7.2 \mathrm{~Hz}, 3 \mathrm{H}), 3.17(\mathrm{~d}, J=7.2 \mathrm{~Hz}$, 2H), $3.40(\mathrm{~s}, 3 \mathrm{H}), 4.57(\mathrm{~s}, 2 \mathrm{H}), 7.54(\mathrm{~d}, J=8.0 \mathrm{~Hz}, 1 \mathrm{H}), 7.92-7.95(\mathrm{~m}, 1 \mathrm{H}), 8.49(\mathrm{~d}, J=2.0 \mathrm{~Hz}, 1 \mathrm{H})$ ppm; ${ }^{13} \mathrm{C}-\mathrm{NMR}\left(\mathrm{DMSO}-d_{6}\right): \delta 165.1,150.7,150.5,140.9,131.8,124.8,118.2,51.9,46.8,36.1,13.9$; HRMS (EI+) calcd for $\mathrm{C}_{12} \mathrm{H}_{14} \mathrm{ClN}_{5} \mathrm{O}_{2} \mathrm{~S}\left(\mathrm{M}^{+}\right)$: 327.0557 . Found: 327.0559 .

N-((6-Chloropyridin-3-yl)methyl)-2-imino-3-methyl-5-nitro-2,3-dihydrothiazol-4-amine (22). Yield: 82\%, m.p.: 116.4-118.4 ${ }^{\circ} \mathrm{C} ;{ }^{1} \mathrm{H}-\mathrm{NMR}\left(\mathrm{DMSO}_{6}\right): \delta 3.28(\mathrm{~s}, 3 \mathrm{H}), 5.37$ (s, 2H), 7.80 (s, 1H) ppm; ${ }^{13} \mathrm{C}-\mathrm{NMR}\left(\mathrm{DMSO}-d_{6}\right): \delta 152.1,142.5,141.2,42.5,34.6,33.3$; HRMS (ES-) calcd for $\mathrm{C}_{8} \mathrm{H}_{8}{ }^{35} \mathrm{ClN}_{5} \mathrm{O}_{2} \mathrm{~S}_{2}$ $(\mathrm{M}-\mathrm{H})^{+}$: 303.9808. Found: 303.9716. calcd for $\mathrm{C}_{8} \mathrm{H}_{8}{ }^{37} \mathrm{ClN}_{5} \mathrm{O}_{2} \mathrm{~S}_{2}(\mathrm{M}-\mathrm{H})^{+}:$: 305.9778. Found: 305.9703.

$\mathrm{N}$-((6-Chloropyridin-3-yl)methyl)-3-ethyl-2-imino-5-nitro-2,3-dihydrothiazol-4-amine (23). Yield: 78\%, m.p.: $115.4-117.2{ }^{\circ} \mathrm{C} ;{ }^{1} \mathrm{H}-\mathrm{NMR}$ (DMSO- $\left.d_{6}\right): \delta 1.23(\mathrm{t}, J=7.2 \mathrm{~Hz}, 3 \mathrm{H}), 3.66(\mathrm{q}, J=7.2 \mathrm{~Hz}, 2 \mathrm{H}), 5.34$ $(\mathrm{s}, 2 \mathrm{H}), 7.81(\mathrm{~s}, 1 \mathrm{H}) ;{ }^{13} \mathrm{C}-\mathrm{NMR}\left(\mathrm{DMSO}-d_{6}\right): \delta 152.1,141.2,134.7,42.7,42.1,15.7$; HRMS (ES-) calcd for $\mathrm{C}_{9} \mathrm{H}_{10}{ }^{35} \mathrm{ClN}_{5} \mathrm{O}_{2} \mathrm{~S}_{2}(\mathrm{M}-\mathrm{H})^{+}:$317.9964. Found: 317.9870 . calcd for $\mathrm{C}_{9} \mathrm{H}_{10}{ }^{37} \mathrm{ClN}_{5} \mathrm{O}_{2} \mathrm{~S}_{2}(\mathrm{M}-\mathrm{H})^{+}$: 319.9935. Found: 319.9836.

\subsection{Biological Assay}

All compounds were dissolved in acetone and diluted with water containing Triton X-100 $\left(0.1 \mathrm{mg} \mathrm{L}^{-1}\right)$ to obtain series concentrations of 500.0, 250.0, $125.0 \mathrm{mg} \mathrm{L}^{-1}$ and others for bioassays. Pea aphids (Aphis craccivora) were dipped according to our previously reported procedure [13,23]. Tender shoots of soybean with 40-60 healthy apterous adults were dipped in diluted solutions of the chemicals containing Triton X-100 $\left(0.1 \mathrm{mg} \mathrm{L}{ }^{-1}\right)$ for $5 \mathrm{~s}$, the superfluous fluid was removed, and the shoots were placed in the conditioned room $\left(25 \pm 1{ }^{\circ} \mathrm{C}, 50 \% \mathrm{RH}\right)$. Water containing Triton $\mathrm{X}-100\left(0.1 \mathrm{mg} \mathrm{L}{ }^{-1}\right)$ was used as control. Mortality was assessed after $24 \mathrm{~h}$. Each treatment had three repetitions, and the data were adjusted and subjected to probit analysis as before.

\section{Conclusions}

In conclusion, a series of a novel class of neonicotinoids, in which the common nitromethylene pharmacophore was substituted by electrophilic thiocyanogen reagents, were designed and synthesized. 
Most of the compounds exhibited excellent insecticidal activities against cowpea aphids (A. craccivora), which implied that reaction of nitromethylene with the electrophilic system to obtain novel neonicotinoids analogues with high activities was feasible.

\section{Acknowledgments}

The work was financial supported by National Basic Research Program of China (973 Program, 2010CB126100), National High Technology Research Development Program of China (863 Program, 2011AA10A207), Key Projects in the National Science \& Technology Pillar Program during the Twelfth Five-Year Plan Period (2011BAE06B05). National Natural Science Foundation of China (21002030), Natural Science Foundation of Shanghai (10ZR1407300), Shanghai Education Committee (12ZZ057) and the Fundamental Research Funds for the Central Universities. This work was also partly supported Shanghai Leading Academic Discipline Project, Project Number: B507 and Australia DC Foundation.

\section{References}

1. Ohno, I.; Tomizawa, M.; Aoshima, A.; Kumazawa, S.; Kagabu, S. Trifluoroacetyl neonicotinoid insecticides with enhanced hydrophobicity and effectiveness. J. Agric. Food Chem. 2010, 58, 4999-5003.

2. Tomizawa, M.; Casida, J.E. Selective toxicity of neonicotinoids attributable to specificity of insect and mammalian nicotinic receptors. Annu. Rev. Entomol. 2003, 48, 339-364.

3. Tomizawa, M.; Casida, J.E. Neonicotinoid insecticide toxicology: Mechanisms of selective action. Annu. Rev. Pharmacol. Toxicol. 2005, 45, 247-268.

4. Matsuda, K.; Shimomura, M.; Ihara, M.; Akamatsu, M.; Sattelle, D.B. Neonicotinoids show selective and diverse actions on their nicotinic receptor targets: Electrophysiology, molecular biology, and receptor modeling studies. Biosci. Biotechnol. Biochem. 2005, 69, 1442-1452.

5. Jeschke, P.; Nauen, R. Neonicotinoids-From zero to hero in insecticide chemistry. Pest Manag. Sci. 2008, 64, 1084-1098.

6. Yu, H.; Qin, Z.; Fang, J. Synthesis and insecticidal activity of N-substituted (1,3-thiazole)alkyl sulfoximine derivatives. J. Agric. Food Chem. 2008, 56, 11356-11360.

7. Unruh, T.; Willett, L. Survey for resistance to four insecticides in Myzus persicae clones from peach trees and weeds in south-central Washington. J. Econ. Entomol. 2008, 101, 1919-1926.

8. Soderlund, D.M. Pyrethroids, knockdown resistance and sodium channels. Pest Manag. Sci. 2008, 64, 610-616.

9. Sayyed, A.H.; Ahmad, M.; Saleem, M.A. Cross-resistance and genetics of resistance to indoxacarb in Spodoptera litura (Lepidoptera: Noctuidae). J. Econ. Entomol. 2008, 101, 472-479.

10. Elbert, A.; Nauen, R. Resistance of Bemisia tabaci (Homoptera: Aleyrodidae) to insecticides in southern Spain with special reference to neonicotinoids. Pest Manag. Sci. 2000, 56, 60-64.

11. Rauch, N.; Nauen, R. Identification of biochemical markers linked to neonicotinoid cross resistance in Bemisia tabaci (Hemiptera: Aleyrodidae). Arch. Insect Biochem. Physiol. 2003, 54, $165-176$. 
12. Nauen, R.; Denholm, I. Resistance of insect pests to neonicotinoid insecticides: Current status and future prospects. Arch. Insect Biochem. Physiol. 2005, 58, 200-215.

13. Tian, Z.; Shao, X.; Li, Z.; Qian, X.; Huang, Q. Synthesis, insecticidal activity, and QSAR of novel nitromethylene neonicotinoids with tetrahydropyridine fixed cis configuration and exo-ring ether modification. J. Agric. Food Chem. 2007, 55, 2288-2292.

14. Shao, X.; Li, Z.; Qian, X.; Xu, X. Design, synthesis, and insecticidal activities of novel analogues of neonicotinoids: Replacement of nitromethylene with nitroconjugated system. J. Agric. Food Chem. 2009, 57, 951-957.

15. Zhang, W.; Yang, X.; Chen, W.; Xu, X.; Li, L.; Zhai, H.; Li, Z. Design, multicomponent synthesis, and bioactivities of novel neonicotinoidanalogues with 1,4-dihydropyridine scaffold. J. Agric. Food Chem. 2010, 58, 2741-2745.

16. Shao, X.; Fu, H.; Liu, Z.; Xu, X.; Li, Z. Divalent and oxabridged neonicotinoids constructed by dialdehydes and nitromethylene analogues of imidacloprid: Design, synthesis, crystal structure, and insecticidal activities. J. Agric. Food Chem. 2010, 58, 2696-2702.

17. Tomizawa, M.; Zhang, N.J.; Durkin, K.A.; Olmstead, M.M.; Casida, J.E. The eonicotinoid electronegative pharmacophore plays the crucial role in the high affinity and selectivity for the drosophila nicotinic receptor: An anomaly for the nicotinoid cation- $\pi$ interaction model. Biochemistry 2003, 42, 7819-7827.

18. Babine, R.E.; Bender, S.L. Molecular recognition of protein-ligand complexes: Applications to drug design. Chem. Rev. 1997, 97, 1359-1472.

19. Kagabu, S.; Matsuno, H. Chloronicotinyl insecticides. 8. Crystal and molecular structures of imidacloprid and analogous compounds. J. Agric. Food Chem. 1997, 45, 276-281.

20. Tomizawa, M.; Lee, D.L.; Casida, J.E. Neonicotinoid insecticides: Molecular features conferring selectivity for insect versus mammalian nicotinic receptors. J. Agric. Food Chem. 2000, 48, 6016-6024.

21. Tokumitsu, T.; Hayashi, T. Reaction of .beta.-nitroenamines with electrophilic reagents. Synthesis of .beta.-substituted .beta.-nitroenamines and 2-imino-5-nitro-4-thiazolines. J. Org. Chem. 1985, 50, $1547-1550$.

22. Tian, Z.Z.; Jiang, Z.X.; Li, Z.; Song, G.H.; Huang, Q.C. Syntheses and biological activities of octahydro-1H-cyclopenta[d]pyrimidine derivatives. J. Agric. Food Chem. 2007, 55, 143-147.

23. Srinwasachari, R. Nitroenmines: Preparation, structure and synthetic potential. Tetrahedron 1981, $37,1453-1480$.

24. Mattson, A.E.; Zuhl, A.M.; Reynolds, T.E.; Schiedt, K.A. Direct nucleophilic acylation of nitroalkenes promoted by a fluoride anion/thiourea combination. J. Am. Chem. Soc. 2006, 128, 4932-4933.

25. Coustard, J.M.; Soro, Y.; Siaka, S.; Bamba, F.; Cousson, A. Synthesis of 6- to 10-membered ring (E)-hydroxyiminohydroazaazoniabenzocycloalkenes derivative from cyclization of 2-nitromethylene-1-( $\omega$-phenylalkyl)imidazolidine or 2-nitromethylene-1-( $\omega$-phenylalkyl) hexahydropyrimidine in trifluoromethanesulfonic acid. Tetrahedron 2006, 62, 3320-3328.

26. Zhang, N.J.; Tomizawa, M.; Casida, J.E. $\alpha$-Nitro Ketone as an electrophile and nucleophile: Synthesis of 3-Substituted 2-nitromethylenetetrahydrothiophene and-tetrahydrofuran as Drosophila nicotinic receptor probes. J. Org. Chem. 2004, 69, 876-881. 
27. Foks, H.; Pancechowska-Ksepko, D.; Janowiec, M.; Zwolska, Z.; Augustynowicz-Kopeé, E. Synthesis and tuberculostatic activity of some 1,1-bis-methylthio-2-nitro-ethene derivatives. Phosphorus Sulfur Silicon Relat. Elem. 2005, 180, 2291-2297.

Sample Availability: Samples of the title compounds are available from the authors.

(C) 2012 by the authors; licensee MDPI, Basel, Switzerland. This article is an open access article distributed under the terms and conditions of the Creative Commons Attribution license (http://creativecommons.org/licenses/by/3.0/). 